\title{
Analysis of the sleep quality of elderly people using biomedical signals
}

\author{
L. Moreno-Alsasua, B. Garcia-Zapirain and A. Mendez-Zorrilla* \\ DeustoTech-Life Unit, University of Deusto, Avda. Universidades, 24. 48007, Bilbao, Spain
}

\begin{abstract}
This paper presents a technical solution that analyses sleep signals captured by biomedical sensors to find possible disorders during rest. Specifically, the method evaluates electrooculogram (EOG) signals, skin conductance (GSR), air flow (AS), and body temperature. Next, a quantitative sleep quality analysis determines significant changes in the biological signals, and any similarities between them in a given time period. Filtering techniques such as the Fourier transform method and IIR filters process the signal and identify significant variations. Once these changes have been identified, all significant data is compared and a quantitative and statistical analysis is carried out to determine the level of a person's rest. To evaluate the correlation and significant differences, a statistical analysis has been calculated showing correlation between EOG and AS signals $(\mathrm{p}=0,005), \mathrm{EOG}$, and GSR signals $(\mathrm{p}=0,037)$ and, finally, the EOG and Body temperature $(\mathrm{p}=0,04)$. Doctors could use this information to monitor changes within a patient.
\end{abstract}

Keywords: EOG, GSR, AS, body temperature, sleep quality, processing

\section{Introduction}

This paper presents the design and development of a technological solution that aims to solve problems during rest and detect the level of sleep quality. In doing so, it aids in the diagnostic process and allows doctors to monitor the progress of patients.

Sleep is not a uniform process, which is why the studies are carried out from when the subjects fall asleep until they wake up. In this paper, we acquired electrooculogram (EOG) signals, body temperature, Air flow Sensor (AS), and skin conductance (GSR) to monitor subjects and analyze their sleep quality.

When a lack of sleep occurs over a period of time, sleep disorders emerge. These affect the normal development of the sleep-wake cycle, meaning that phases of sleep may suffer disturbances [1]. If these modifications occur over a long period of time, they can cause health problems and interfere with physical, mental, and emotional functionality. This phenomenon is called sleeping sickness or a sleep disorder. The most significant disturbances are insomnia (suffered by $15-30 \%$ of the population, $10 \%$ chronically), apnea ( $7 \%$ of the population suffer from this and $85 \%$ of them go undiagnosed), narcolepsy (affecting $0.06 \%$ ) and parasomnias (10-20\% of the population).

\footnotetext{
${ }^{*}$ Address for correspondence: A. Mendez-Zorrilla, DeustoTech-Life Unit, University of Deusto, Avda. Universidades, 24. 48007, Bilbao, Spain. Tel.: +34 944139000; Fax: 944-456-817; E-mail: amaia.mendez@deusto.es.
}

0959-2989/15/\$35.00 @ 2015 - IOS Press and the authors. 
In short, designing and developing a technological solution for sleep quality is presented by analyzing the patient's physiological signals. This involves identifying changes in sleep by processing the signals in which certain parameters are analyzed to obtain a quantitative evaluation of sleep quality. In doing so, an accurate diagnosis can be made, which will also enable doctors to monitor treatment progress.

The well-established techniques considered for analyzing sleep quality are polysomnography, respiratory polygraphy, testing for multiple sleep latencies, and the video-EEG dream record [2]. These techniques are typically only performed at a hospital because they are difficult and expensive. This paper offers an alternative sleep monitoring solution, which can be used at patients' homes at a lower cost.

Many papers have analyzed sleep disorders with different types of sensors, like EEG with EOG [3] or air flow with GSR [4]. However, there are not any published papers that use EOG, body temperature, AS, and GSR to analyze sleep disorders.

\section{Methods}

This section describes the methods used to develop this process, which allowed us to establish a sleep monitor system and evaluate its quality.

\subsection{Participants}

Five people had their sleep monitored; two male and three female. Two females and one male were between the ages of 18 and 25; there was also 50-year-old female subject and a 55-year-old male subject. The recordings were two hours in length, which is long enough to detect a complete 90 minute sleep cycle. Individuals suffering from chronic illnesses which may alter the paper's results were excluded. There are not enough samples to make conclusions about the impact of age and gender on sleep quality. This purpose of this study is to guarantee the effectiveness of the sensors and the method. The sensors are attached to specific points to enable precise data collection. The EOG signal is obtained by three sensors: the reference sensor is attached behind one ear and the other two above the eyebrows. The nasal air flow sensor is attached just below the nose. Lastly, the GSR and temperature sensors are attached to the same hand.

The signals from the sensors are captured and processed to analyze sleep quality. The subjects attached the sensors in their homes without any specialist help. This experiment passed the ethical committee of the University of Deusto, and the patients signed an informed consent form.

\subsection{Evaluation assessment}

As part of the evaluation, the subjects completed two questionnaires. The first is the ESS (Epworth Sleepiness Scale) [5], which is a questionnaire assessing the propensity to fall asleep in 8 different, sedentary situations. The second is the PSQI (Pittsburgh Sleep Quality Index) [6], which collects information from the patient. The following table shows the results obtained from both questionnaires.

From the answers obtained from Table 1, each questionnaire's total score is calculated. This final calculation determines the final result of both questionnaires. Table 2 shows the final scores from each subject. 
Table 1

Responses from the sleep questionnaires

\begin{tabular}{|c|c|c|c|c|c|}
\hline \multicolumn{6}{|l|}{ ESS Questionnaire } \\
\hline & Subject 1 & Subject 2 & Subject 3 & Subject 4 & Subject 5 \\
\hline Question 1 & 0 & 3 & 1 & 1 & 0 \\
\hline Question 2 & 1 & 3 & 1 & 0 & 1 \\
\hline Question 3 & 0 & 2 & 1 & 0 & 1 \\
\hline Question 4 & 1 & 3 & 0 & 0 & 0 \\
\hline Question 5 & 2 & 3 & 3 & 1 & 2 \\
\hline Question 6 & 0 & 2 & 0 & 0 & 0 \\
\hline Question 7 & 0 & 2 & 1 & 1 & 1 \\
\hline Question 8 & 0 & 2 & 0 & 0 & 0 \\
\hline \multicolumn{6}{|l|}{ PSQI Assessment } \\
\hline Subjective sleep quality & 2 & 1 & 2 & 2 & 1 \\
\hline Sleep latency & 1 & 1 & 1 & 2 & 1 \\
\hline Sleep duration & 0 & 2 & 2 & 1 & 2 \\
\hline Normal sleep efficiency & 0 & 0 & 0 & 0 & 0 \\
\hline Sleep disturbances & 1 & 3 & 1 & 3 & 2 \\
\hline Use of medication in order to sleep & 2 & 1 & 2 & 2 & 1 \\
\hline Daytime dysfunction & 2 & 2 & 1 & 1 & 2 \\
\hline
\end{tabular}

Table 2

Questionnaire results

\begin{tabular}{|l|l|l|l|l|l|}
\hline Total Score & Subject 1 & Subject 2 & Subject 3 & Subject 4 & Subject 5 \\
\hline ESS & 4 & 20 & 7 & 3 & 6 \\
\hline PSQI & 8 & 10 & 9 & 11 & 9 \\
\hline
\end{tabular}

On the ESS, if the score is lower than 6 points, diurnal sleepiness is low or absent; if it is between 7 and 8 , it is the average for the population; if the score is over 9, sleepiness is excessive and a specialist should be consulted. In the PSQI, the total sum of each item can vary from 0 to 21 points. The cut-off point would be 5, where it would be considered that the subjects enjoy good sleep quality.

\subsection{Acquisition of data}

The EOG, GSR, AS, and body temperatures of subjects were monitored to acquire the data. EOG signal monitoring is obtained using an EMG sensor, which measures the patient's muscular movement. Its readings are based on the voltage difference existing between the cornea and retina. Under normal conditions, the difference is 0.4 to $5 \mathrm{mV}$. By attaching electrodes close to the eye muscles, eye movement is measured to obtain EOG signals. These signals are extremely useful to detect the NREM (Non- Rapid Eye Movement) phase when eye movement can barely be detected, and the REM (Rapid Eye Movement) phase when movement accelerates significantly.

The AS (airflow signal) is a nose and mouth flow sensor that detects the flow of nasal and oral air during sleep. It records changes when the patient breathes in and out, which is essential to detect apnea and hypopnea disorders. This signal is obtained with a $200 \mathrm{~Hz}$ minimum sampling frequency. To detect changes in the signal, spectral information is extracted in bands from $0.1 \mathrm{~Hz}$ to $15 \mathrm{~Hz}$.

The GSR (Galvanic Skin Response) records the skin's electrical conductance and resistance. This biomedical signal depends on the skin's humidity caused by sweating. Bodily secretions are controlled by the SNS (sympathetic nervous system), so skin conductance is a good indicator of physiological 
and psychological arousal $[7,8]$. Processing this signal identifies the sudden change from deep sleep to a shallower phase, which may or not lead to waking up.

Lastly, body temperature is a very stable, endogenous marker and is therefore useful in studying circadian disorders. Body temperature follows a 24-hour rhythm: it starts to drop at the beginning of the sleep period, it reaches its lowest point in the small hours, and then rises in the early morning coinciding with waking up $[9,10]$. This variation in temperature between sleep/wakefulness is $1{ }^{\circ} \mathrm{C}$. Values are regarded as normal if the amplitude (difference between the maximum peak and minimum peak) is $\geq 0.7^{\circ} \mathrm{C}$ and it is in phase with the set sleep schedule.

\subsection{System design}

This section specifies the reliable sleep monitoring system. The functions of each subsystem are described in Table 3. This section splits into two subcategories: Hardware and Software, with each further divided into sub-blocks. Data obtained from these sensors is pre-processed with Arduino software to obtain accurate data.

\subsubsection{Hardware}

Hardware is composed of two sub-blocks: storing data obtained from biomedical sensors, and data pre-processing used for the optimal adaptation of the signals.

Figure 1 shows how the first block works. The input is non-pre-processed physiological signals and the output sent to the PC is modified signals for subsequent analysis. The following sections provide a more detailed description.

Table 3

System functions

\begin{tabular}{|l|l|}
\hline Subsystem & Functions \\
\hline Sensors & $\begin{array}{l}\text { Monitoring physiological parameters. } \\
\text { Accessing data. }\end{array}$ \\
\hline Data processing & $\begin{array}{l}\text { Means of communication between the circuit and the software. } \\
\text { Adapting data. } \\
\text { Designing algorithms for data processing corresponding to the signal type. }\end{array}$ \\
\hline Data analysis & $\begin{array}{l}\text { Recording the subjects. } \\
\text { Collecting significant data from each signal type. } \\
\text { Comparing significant data between the signals occurring at adjoining moments in time. } \\
\text { Statistical calculation. }\end{array}$ \\
\hline Display & $\begin{array}{l}\text { Displaying processed signals. } \\
\text { Displaying changes in common between signals. }\end{array}$ \\
\hline
\end{tabular}

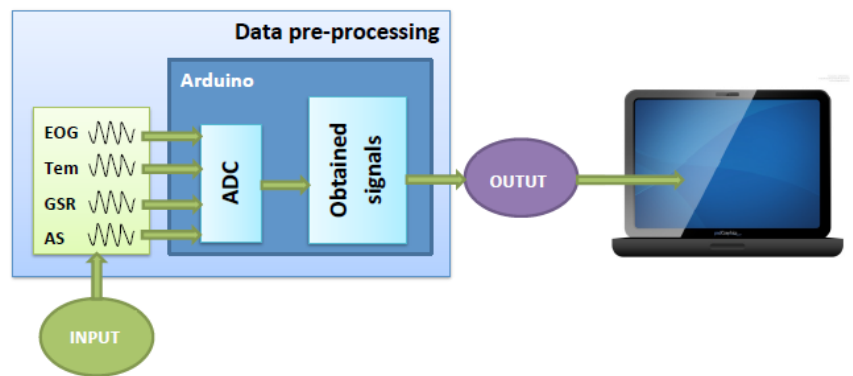

Fig. 1. Diagram of the hardware block. 


\subsubsection{Software}

The software is composed of three elements: data processing, data analysis, and displaying results. This block collects pre-processed signals by Arduino, and processes each signal type appropriately: filtering the signal, and analyzing it in time and frequency. Filtering the signal is essential to obtain a clear signal. To do so, each signal needs a different filtering process. EOG filtering needs a $0.3 \mathrm{~Hz}$ high-pass filter and a $15 \mathrm{~Hz}$ low-pass filter. AS filtering need a $25 \mathrm{~Hz}$ low pass filter.

Once processed, the stored data is analyzed by processing algorithms, which conduct a quantitative analysis of the signals so that they may detect possible disturbances during sleep. The algorithms include a comparative of the EOG signal with AS, body temperature, and GS signals to provide a relation with the change in signals and sleep disturbance.

Furthermore, a statistical calculation is made to ensure effective monitoring and to validate the results. A statistical analysis determines the agreement between the signals' changes and how they affect the patient's sleep quality. This next step is to calculate changes in the signals to determine patterns and discrepancies.

The third sub-block can be seen in Figure 2. The data is displayed using a user's interface to facilitate the diagnosis made by the medical expert. This process is shown in Figure 2.

\section{Results}

This section compiles the results obtained from each patient to prove that the system works properly. Throughout this study, each signal's most significant points have been recorded: AS peaks, EOG peaks, temperature change, resistance jump, and conductance jump. Monitoring the most unstable points was then carried out to determine whether such points condition the other signals or not. The final results are obtained via the user's interface, where the processing algorithms are compiled and each subject's characteristics analyzed.

\subsection{Results obtained via the interface}

Analysis of the signals was conducted using the processed EOG (see Figure 3), GSR, AS (see Figure 4) signals, and body temperature. Specific filtering was carried out on each signal, to prevent the signals from being affected by external interferences. EOG filtering, for instance, needs a $0.3 \mathrm{~Hz}$ highpass filter and a $15 \mathrm{~Hz}$ low-pass filter. Figure 5 shows how filtering helps to interpret the signal. The following figure displays an unfiltered EOG type signal after processing.

Once the signal has been filtered, its most significant peaks are displayed and marked with a circle. Unstable points and the times when they occur are stored to later compare them with other signals. The following figure compares the EOG signal with the AS signal. The latter requires the nasal flow speed to be calculated to detect the unstable points when the subject breathed irregularly.

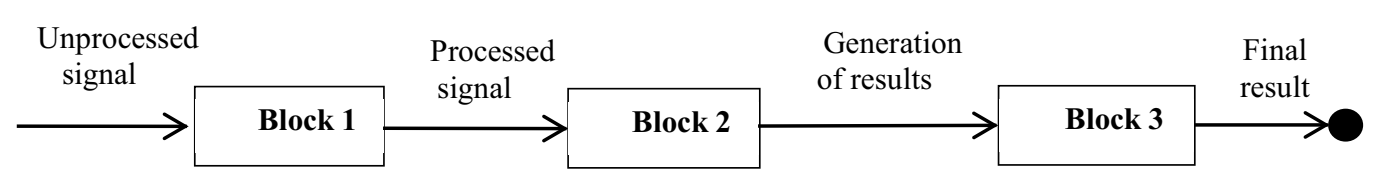

Fig. 2. Diagram of the Software block. 

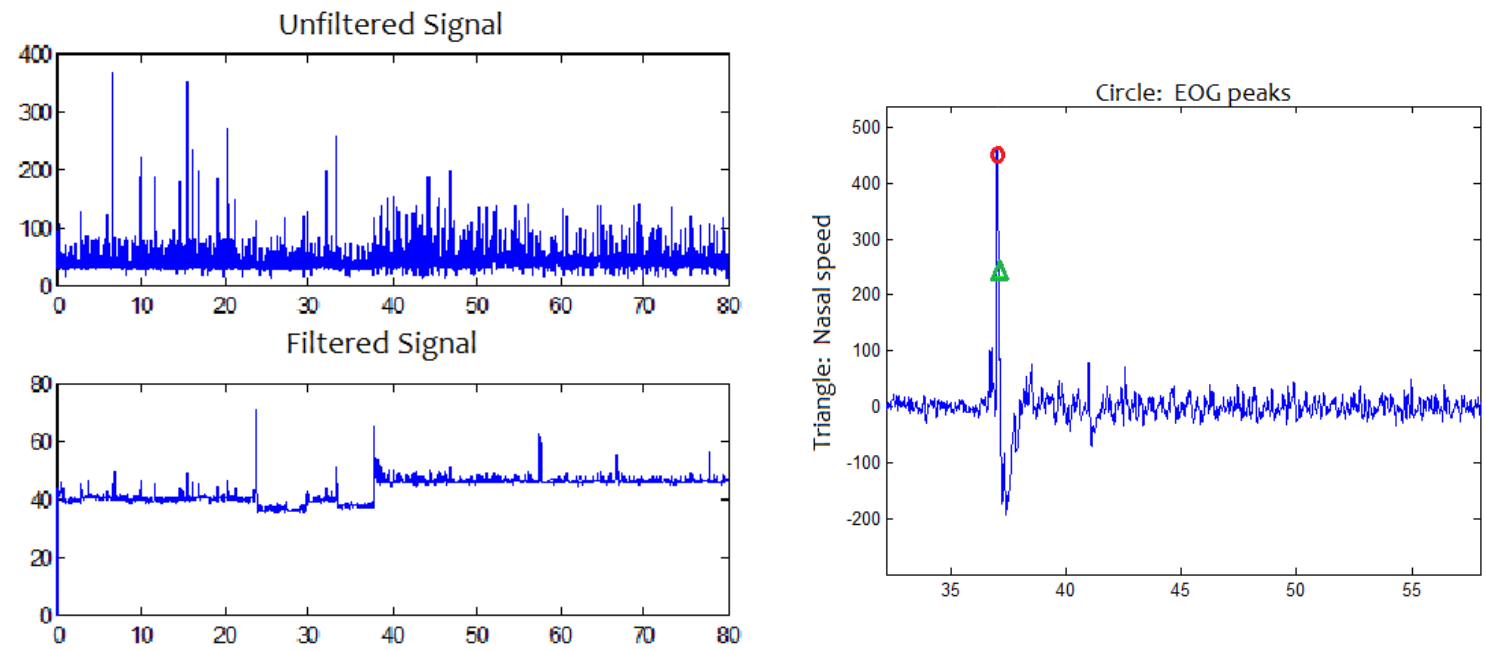

Fig. 3. EOG signal.

Fig. 4. EOG and AS comparison.

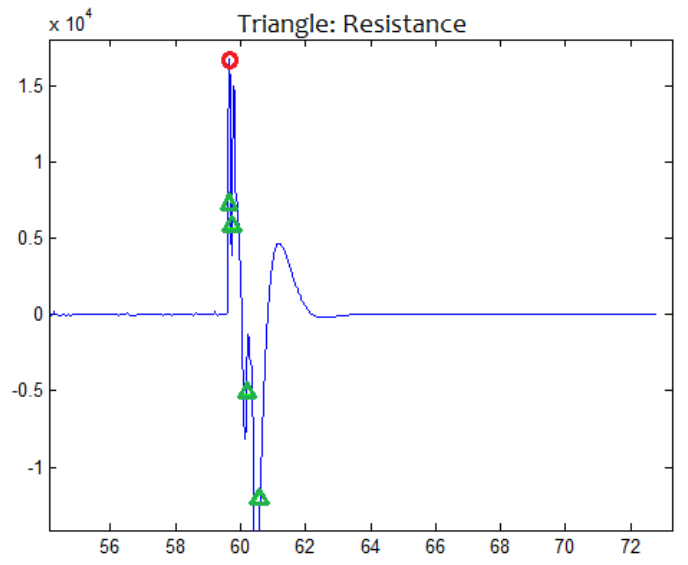

Fig. 5. EOG, GSR and temperature comparison.

Furthermore, body temperature and GSR are directly related: if skin conductance rises, body temperature falls [11]. To obtain points of agreement between both signals, the number of changes for each metric must be detected, and then critical moments for each metric must be compared. The data occurring in common between GSR and body temperature is stored for subsequent comparison with the EOG signal.

Unstable points between the EOG, GSR, and body temperature are compared to determine if they relate to the EOG signal. If a change occurred at the same time as an EOG signal, the changes are thought to have conditioned the subject's rest.

\subsection{Statistical analysis}

To validate the study, a statistical analysis is conducted determining the agreement between the signals' changes and how they affect the patient's sleep quality. This study calculates the measurements of 
the signal's significant changes to determine how the data groups and/or scatters. Each subject's descriptive statistics are calculated, which include the mean, standard deviation (SD), and each value's maximum and minimum. SD quantifies variation or dispersion of a set of data values [12]. A standard deviation close to 0 indicates that the data points tend to be very close to the mean of the set, while a high standard deviation indicates that the data points are spread out over a wider range of values.

With this data, Spearman's rank correlation coefficient is then calculated. This correlation is a nonparametric measure of statistical dependence between two variables. It assesses how well the relationship between two variables can be described using a monotonic function [13]. In this case, the variables are the number of peaks in one signal, like EOG, and jumps in the others, like body temperature. The following graph shows the significant changes $(\mathrm{p}<0.05)$ obtained, and their level of correlation.

Figure 6 shows data close to 1 . This data has a very strong positive correlation, i.e. if a value increases or decreases, the other will also. However, values close to -1 have a very strong negative correlation; if a value increases, the other decreases, and vice versa. This analysis proves the effectiveness of the Arduino and 'e-Health' shield to obtain a clear data.

\section{Discussion and conclusions}

This section discusses the results and draws conclusions from the data.

This system efficiently monitors subjects' sleep in their homes. This system can therefore help diagnose sleep disorders [14], and sleep studies can be conducted without the need to involve specialized clinics. The most significant conclusions and discussions concerning each stage are summarized below, as well as the results obtained in the process.

The statistical correlation performed using Spearman's rho indicates that the data collected produces significant statistical results on up to eleven occasions. Therefore, the changes occurring in the EOG signal, in common with the other signals' disturbances, are related. Thus, if a large number of changes in common between the signals are obtained, the user's level of rest is lower. The changes in common occurring between the EOG signal and the AS signal have produced significant data $(\mathrm{p}=0.005<0.05)$

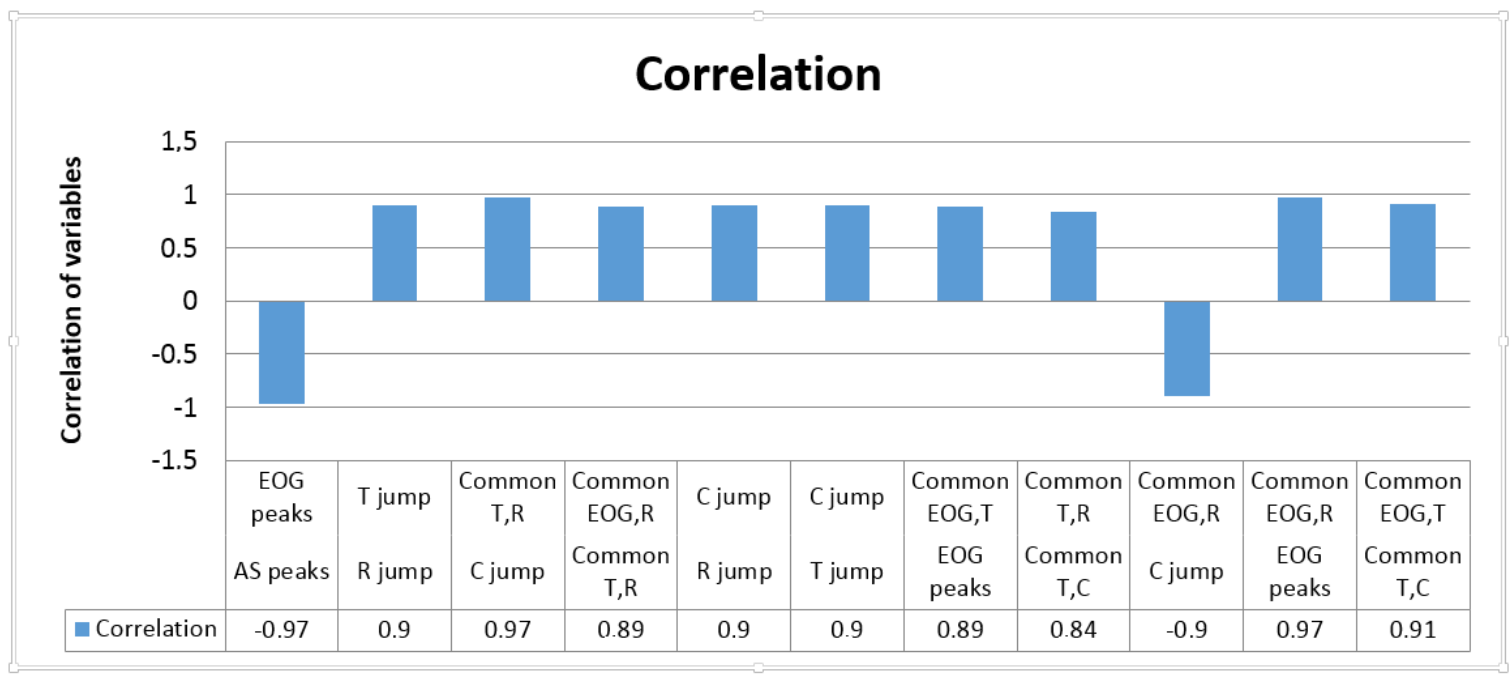

Fig. 6. Correlation of variables. 
with a 0.97 correlation out of 1 . This is in line with what is described in studies of sleep apnea $[15,16]$, since unstable moments in breathing have been found to coincide with the EOG signal and indicating that the subject's rest has been affected.

Regarding different studies [17, 18], each signal's significant points have been located and these points have also been related to the others to obtain a direct relationship between the subject's level of rest and the number of changes recorded during the recording period. High-value changes in common have also been detected between temperature and GSR. This data, together with the data in common with EOG, complements the results obtained in relation to temperature during sleep [19]. Similarly, significant changes in common have been obtained with EOG and GSR $(p=0.037<0.05)$ and in EOG and body temperature $(\mathrm{p}=0.04<0.05)$.

To develop the system, the signals obtained via hardware are adapted, and conflicts are avoided in interpretation and subsequent processing. If the signals obtained by the "e-Health" sensor are not recorded correctly, data interpretation can vary. In this study, the readings of each subject's physiological activities have been successfully and appropriately obtained, which aids data interpretation via software.

With regard to processing, each signal (EOG, GSR, AS, and body temperature) has been analyzed to adapt each one appropriately. Fourier frequency analysis aids in the detection of conflicting frequencies; IIR filtering eliminated them. If such filtering fails to be done properly, it can result in errors in reading the data.

By analyzing the significant changes experienced by each subject, it can be seen that there is a certain relationship between the changes occurring at moments in time close to the subject's level of rest. It has been observed that the number of jumps in conductance and resistance, and the changes arising from temperature do not always have a direct relationship with the subject's rest. The relationship arises when the changes coincide with the EOG signal. Furthermore, it can be seen that the air flow signal suffers greater variation if the EOG signal displays a larger number of disturbances. Moreover, it has been noted that, on days when the subject has reached the REM phase, the number of coincidences between signals is better in relation to the days when they have failed to reach this phase. It is therefore thought that these are the days when a higher level of sleep quality is achieved.

\section{Acknowledgment}

This project has been partially funded under the grant IG-2014/01149 (h-SEAT project) and Basque Government Department of Universities and Research. The authors also wish to thank to the companies Bilbomatica, DxD and Zuentzat for their support.

\section{References}

[1] R.M. Chemelli, J.T. Willie, C.M. Sinton, J.K. Elmquist, T. Scammell, C. Lee and M. Yanagisawa, Narcolepsy in orexin knockout mice: Molecular genetics of sleep regulation, Cell 98 (1999), 437-451.

[2] I.G. de Gurtubay, Estudios diagnósticos en patología del sueño Diagnosis of sleep disorders, Anales del Sistema Sanitario. de. Navarra 30 (2007).

[3] H.W. Agnew, W.B. Webb and R.L. Williams, The first night effect: An EEG study of sleep, Psychophysiology 2 (1966), 263-266.

[4] M.W. Johns, B.A. Cornell and J.P. Masterton, Monitoring sleep of hospital patients by measurement of electrical resistance of skin, Journal of Applied Physiology 27 (1969), 898-901.

[5] M.W. Johns, A new method for measuring daytime sleepiness: The Epworth sleepiness scale, Sleep 14 (1991), $540-545$. 
[6] Interpretación del índice de calidad del sueño de Pittsburgh, available at: http://investigacionencuidados.es/syce/pdf/pittsburg.pdf, acessed: Jul. 25, 2014.

[7] L.C. Johnson and A. Lubin, Spontaneous electrodermal activity during waking and sleeping, Psychophysiology 3 (1966), 8-17.

[8] G. Noll, M. Elam, M. Kunimoto, T. Karlsson and B.G. Wallin, Skin sympathetic nerve activity and effector function during sleep in humans, Acta Physiologica Scandinavica 151 (1994), 319-329.

[9] M. Uchiyama, M. Okawa, K. Shibui, K. Kim, H. Tagaya, Y. Kudo, K. Takahashi et al., Altered phase relation between sleep timing and core body temperature rhythm in delayed sleep phase syndrome and non-24-hour sleep-wake syndrome in humans, Neuroscience Letters 294 (2000), 101-104.

[10]E.D. Weitzman, C. Nogeire, M. Perlow, D. Fukushima, J. Sassin, P. Mcgregor, L. Hellman et al., Effects of a prolonged 3-hour sleep-wake cycle on sleep stages, plasma cortisol, growth hormone and body temperature in man, The Journal of Clinical Endocrinology \& Metabolism 38 (1974), 1018-1030.

[11] M.E. Crosby, B. Auernheimer, C. Aschwanden and C. Ikehara, Physiological data feedback for application in distance education, Proceedings of the 2001 workshop on Perceptive user interfaces, ACM, 2001, pp. 1-5.

[12] J.M. Bland and D.G. Altman, Statistics notes: Measurement error, British Medical Journal 313 (1996), 744.

[13] W. Pirie, Spearman rank correlation coefficient, Encyclopedia of Statistical Sciences, 1988.

[14] M.D. Vishesh Kapur, R.E. Sandblom, R. Hert, B. James and D. Sean, The medical cost of undiagnosed sleep apnea, Sleep 22 (1999), 749.

[15] O. Krejcar, J. Jirka and D. Janckulik, Use of mobile phones as intelligent sensors for sound input analysis and sleep state detection, Sensors 11 (2011), 6037-6055.

[16]D.V. Morales, G.G. Hernández, N.M. García and B.C. Mozo, Síndrome de apnea obstructiva del sueño, fisiopatología y diagnóstico, Revista Cubana de Ortodontia 16 (2001), 69-75.

[17]S. Sonnia, M. López and J.R. Sendra Sendra, Apuntes de Bioingeniería, available at: http://www.iuma.ulpgc.es/ jrsendra/Docencia/ElectroMedicina/PDF/Apuntes/ElecMed_ULPGC_2001.pdf

[18] M. Meijide, C. Zamarron, C. Conde, R. Gonzalez-Quintela and A. Gude, Prevalencia de los trastornos respiratorios del sueño y factores asociados, Rey G., Fernandez Aten Primaria 39 (2007), 255-259.

[19] Ch A. Czeisler, et al., Timing of REM sleep is coupled to the circadian rhythm of body temperature in man, Sleep 2.3 (1979), 329-346. 